\title{
Aging, spaced retrieval, and inflexible memory performance
}

\author{
ANTHONY J. BISHARA \\ Indiana University, Bloomington, Indiana \\ AND \\ LARRY L. JACOBY \\ Washington University, St. Louis, Missouri
}

\begin{abstract}
Spaced retrieval is a memory-training technique whereby information is tested at progressively longer delays. Two experiments were conducted in order to examine the effects of spaced retrieval on controlled recollection and automatic influences of memory. In Experiment 1, word pairs were read once, three times, or once and retrieved twice by young and older adults. Retrieval practice improved performance on a later test for both age groups. Experiment 2 was arranged so that recollection opposed automatic influences of retrieval practice. Retrieval practice increased intrusions on a later test only for older adults. Results suggest that because of a deficit in recollection, older adults were less able to oppose the automatic influence of spaced retrieval and thus exhibited less flexible memory performance.
\end{abstract}

A great deal of research has shown that testing improves memory, sometimes even more than does additional studying (see, e.g., Carrier \& Pashler, 1992; Jacoby \& Bartz, 1972; Roediger \& Karpicke, 2006). Repeated testing with progressively increasing delays between tests-termed expanded or spaced retrieval-can greatly enhance memory performance (Landauer \& Bjork, 1978). Spaced retrieval has been used to enhance memory performance in various populations, ranging from Alzheimer's patients (Camp, Foss, O’Hanlon, \& Stevens, 1996; Schacter, Rich, $\&$ Stampp, 1985) to healthy older adults (Logan \& Balota, in press). Despite these successful applications, spaced retrieval may come with the cost of leading to inflexible behavior in populations with deficits in controlled memory functioning, including aging populations.

To illustrate this problem, consider a study in which spaced retrieval was used to train participants with Alzheimer's disease to open a box each time an alarm sounded (Bird \& Kinsella, 1996). Participants did learn to open the box. However, the learning was inflexible and not restricted to the alarm being sounded. For example, 1 participant inappropriately shouted "open the box" the next time she saw the experimenter.

Anecdotes aside, there is currently no published empirical work examining whether spaced retrieval leads to inflexible performance. One published study has examined spaced retrieval in terms of a related issue - a comparison of explicit and implicit memory (Cherry, Simmons, \& Camp, 1999). Unfortunately, spaced retrieval's effects on explicit and implicit memory were ambiguous, because the small sample size prevented statistical hypothesis testing. Closely related to spaced retrieval is the technique of vanishing cues, whereby memory tests are made more difficult by progressively removing cues rather than by progressively increasing test delays (Glisky, Schacter, \& Tulving, 1986). Learning from vanishing cues has been shown to sometimes lead to inflexible behavior (Kapur, Glisky, \& Wilson, 2004).

The present article examines inflexible behavior by comparing spaced retrieval with spaced studying. The results from Experiment 1 suggest that, in comparison with spaced studying, spaced retrieval can improve memory performance for both young and older adults. The results from Experiment 2 suggest that spaced retrieval enhanced both controlled recollection and more automatic influences of memory for young adults. However, recollection is more susceptible to the effects of aging (see, e.g., Jennings \& Jacoby, 1993, 1997); thus, for older adults, spaced-retrieval practice might primarily increase automatic influences, thereby leading to inflexible behavior.

\section{EXPERIMENT 1}

Memory performance in a retrieved condition was compared with that produced by studying word pairs simply by reading them either once or three times (read- $1 \times$ and read $-3 \times$ conditions). For the retrieved condition, we followed Carrier and Pashler (1992) by providing additional exposure to responses when retrieval attempts failed. Doing so is important, because otherwise, failures

A. J. Bishara, abishara@indiana.edu 
to retrieve items on initial tests might hide advantages of retrieval over repeated reading. The retrieved condition involved three trials. On the first trial, associatively related pairs of words (e.g., knee-bone) were presented for study. On the second and third trials, the cue word of the pair was accompanied by a fragment of the response (knee- $\left.b \_n \_\right)$, and participants were instructed to respond with the whole word pair. For the final second of the two trials for which retrieval was attempted, the cue was presented with its intact response (knee-bone), regardless of whether or not retrieval was successful.

An expanded retrieval schedule was used so that there was more intervening time between the second and third trials than between the first and second trials. This expanding schedule was also used for the read- $3 \times$ condition so that it would be comparable. In a later test phase, memory was measured for all three conditions by presenting the cue word along with a fragment of its response (e.g., knee- $b \_n \_$). We expected memory performance to be better in the retrieved condition than in the read- $3 \times$ condition, which in turn was expected to be better than in the read- $1 \times$ condition.

\section{Method}

Participants. Table 1 shows the demographics of participants. The young-adult group consisted of Washington University undergraduates who participated in exchange for either course credit or $\$ 10$. The older-adult group was recruited from the Washington University Psychology Department's older-adult participant pool. Older adults were volunteers from the St. Louis community and participated in exchange for $\$ 10$.

Design and Materials. The two age groups were crossed with three within-subjects conditions: read $1 \times$, read $3 \times$, and retrieved.

A pool of 94 word triplets was selected from norms that were reported by Jacoby (1996). Each triplet included one cue word (e.g., knee) and two associatively related responses (bone, bend) that would complete a word fragment ( $\left.b \_n \_\right)$. Of these triplets, 84 were used as critical items and divided into sets of 28 for the three conditions. The assignment of sets to conditions was counterbalanced across participants. The remaining 10 triplets were used as fillers. In order to reduce serial position effects, two fillers were presented at the beginning and two at the end of the study list. Six filler items were distributed throughout the list in order to obtain the desired repetition schedules.

A distractor list consisted of 84 new, once-presented word pairs (e.g., zoo-cage) that were chosen from association norms (Jacoby, 1996; Nelson, McEvoy, \& Schreiber, 1998). The distractor list inter- vened between the presentation of the study list and its test, and it was meant to reduce recall in order to avoid ceiling effects.

The test list consisted of 84 critical cue words that were presented with a word fragment (e.g., knee- $b_{-} n_{-}$). The fragments were the same as those presented in the retrieved condition. The order of test presentation was random, with the restriction that no more than two items from the same condition could be presented in succession.

Procedure. For the study phase, participants were instructed to read aloud and study word pairs for a later memory test. They were told that some pairs would be repeated with letters missing from the second word (retrieved condition). For these pairs, they were to recall the studied word that completed the fragment and say the whole pair out loud as soon as possible. In the retrieved condition, the complete word pair appeared for $4 \mathrm{sec}$ for the first presentation. On the second and third presentations, the cue word and word fragment (e.g., $k n e e-b \_n \_$) appeared for a maximum of $3 \mathrm{sec}$. As soon as the participant responded — or after $3 \mathrm{sec}$ had elapsed - the word fragment was replaced by the intact pair. The intact pair remained on the screen for $1 \mathrm{sec}$. Participants read the intact pair aloud if they had failed to respond in the previous $3 \mathrm{sec}$. Read- $1 \times$ and read- $3 \times$ word pairs appeared in complete form for $4 \mathrm{sec}$ on each presentation. For read-3 $\times$ and retrieved items, the first presentation was separated from the second by one to two intervening pairs, and the second presentation was separated from the third by five to six intervening pairs.

For the distractor phase, participants were instructed to read aloud and to remember the word pairs for later. Pairs were presented for $2 \mathrm{sec}$ each. In actuality, there was no test for these items.

For the final test phase, participants were tested for their memory of the original study list. Each test trial consisted of a cue word and word fragment (knee-b_n_) appearing on the screen until the participant responded, or for a maximum of $10 \mathrm{sec}$. Participants were instructed to recall the response and to say the whole word pair within that time. They were instructed to give a response even if they had to guess. A blank screen appeared between each trial for $500 \mathrm{msec}$. After the memory test, participants took a computer version of the Shipley vocabulary subtest.

\section{Results and Discussion}

Although lags were chosen to minimize errors during the study phase, errors still occurred occasionally. The probability of producing at least one error out of two opportunities to do so during retrieval practice of an item was lower for young adults $(M=.10, S D=.07)$ than for older adults $(M=.23, S D=.13)[t(34)=3.84, p<.001]$. These errors may strengthen incorrect responses; thus, the main analysis of the final memory test is conditionalized on errorless retrieval during the study phase. For both experiments, unconditionalized data are reported in paren-

Table 1

Demographic Data in Experiments 1 and 2

\begin{tabular}{|c|c|c|c|c|c|c|c|c|c|}
\hline \multirow[b]{2}{*}{ Group } & \multirow[b]{2}{*}{$n$} & \multicolumn{2}{|c|}{ Age } & \multicolumn{2}{|c|}{ Vocab. } & \multicolumn{2}{|c|}{ Health $^{\mathrm{a}}$} & \multicolumn{2}{|c|}{ Education $^{\mathrm{b}}$} \\
\hline & & $M$ & $S D$ & $M$ & $S D$ & Median & Range & Median & Range \\
\hline \multicolumn{10}{|c|}{ Experiment 1} \\
\hline Young adults & 18 & 19.8 & 1.5 & 33.5 & 2.0 & 4 & $3-5$ & 3.0 & $2-4$ \\
\hline Older adults & 18 & $76.0^{* * *}$ & 6.2 & 34.9 & 2.2 & 4 & $2-5$ & $3.0^{*}$ & $1-7$ \\
\hline \multicolumn{10}{|c|}{ Experiment 2} \\
\hline Young adults & 20 & 19.5 & 1.1 & 32.8 & 2.9 & 4 & $3-5$ & 3.0 & $3-3$ \\
\hline Unmatched older adults & 20 & $75.9^{* * *}$ & 5.7 & $35.6^{* *}$ & 3.1 & 4 & $2-5$ & 3.5 & $1-7$ \\
\hline Matched older adults & 20 & $76.0^{* * *}$ & 8.0 & 34.6 & 3.5 & 4 & $1-5$ & 3.0 & $2-7$ \\
\hline
\end{tabular}

Note-Vocab., vocabulary subtest of the Shipley Institute of Living scale. aSelf-rated health, $1=$ poor, $2=$ fair, $3=$ o.k., $4=$ good, $5=$ excellent. ${ }^{b}$ Education levels, $1=$ some high school, $2=$ high school diploma or GED, $3=$ some college, $4=$ B.A. or B.S., $5=$ some postgraduate, $6=$ Master's degree, $7=$ Doctoral degree. Asterisks represent significant differences as compared with young adults in the same experiment, with $t$ tests used for age and vocabulary, and Mann-Whitney $U$ tests used for health and education. ${ }^{*} p<.05 .{ }^{* *} p<.01 .{ }^{* * *} p<.001$. 
theses in tables. Statistical analysis of unconditionalized data is only reported when it changes significance status, and, in most cases, it did not.

Table 2 shows the probability of correct responding on the final test across item type and age. Performance was better for young adults $(M=.83)$ than for older adults $(M=.69)$. When a 2 (age: young and older) $\times 3$ (item type: read $1 \times$, read $3 \times$, and retrieved) mixed ANOVA was performed, there was a significant main effect of age $\left[F(1,34)=24.55, p<.0001, \eta_{\mathrm{p}}^{2}=.42\right]$. Performance was lowest in the read- $1 \times$ condition $(M=.66)$, higher in the read-3 $\times$ condition $(M=.75)$, and highest in the retrieved condition $(M=.92)$. This pattern of results was confirmed by a significant main effect of item type $[F(2,68)=94.03$, $\left.p<.00001, \eta_{\mathrm{p}}^{2}=.73\right]$. Post hoc paired $t$ tests showed that all three conditions were significantly different from one another for both age groups (all $p \mathrm{~s}<.01$ ). The interaction of age and item type was not significant $[F(2,68)=1.53$, $p>.10]$, although it is possible that ceiling effects in the retrieved condition obscured an interaction.

Overall, reading a pair once and then retrieving its response twice produced better memory performance than did studying the pair three times. Although retrieval practice enhanced memory performance, it did not remove the memory disadvantage of older adults.

\section{EXPERIMENT 2}

Retrieval practice might have the automatic influence of increasing the accessibility of responses, as well as a separate effect on controlled recollection. Experiment 2 examined age differences in these two effects of retrieval practice by using an opposition procedure similar to that used by Jacoby (1999) to examine age differences in the effects of repetition.

In Jacoby's experiments, young and older adults read a list of words, with each word being read once, twice, or three times. Next, they heard a second list that they were told to remember. At test, participants were instructed to identify words that they had heard earlier, and they were warned that the test list would include earlier-read words. They were correctly informed that none of the earlier-read words had been presented in the heard list. Repeatedly reading a word was expected to increase its familiarity (an automatic influence of memory) and thus increase the probability that it would be mistakenly judged as heard. However, controlled recollection of having read a word would oppose its familiarity, allowing it to be excluded.

The results showed a significant age $\times$ repetition interaction, suggesting that repetition had two effects. For older adults, repeated reading increased the probability of words being mistakenly accepted as earlier heard, showing the effect of repetition on familiarity. In contrast, young adults were better able to use recollection to oppose familiarity in order to avoid accepting earlier-read words. The age $\times$ repetition interaction suggests that repetition enhanced automatic influences of memory and-at least for young adults - that it also increased controlled recollection that was used to successfully oppose the increased automatic influence.
Table 2

Means and Standard Deviations of the Probability of Correct Recall in Experiment 1

\begin{tabular}{|c|c|c|c|}
\hline \multirow[b]{2}{*}{ Age } & \multicolumn{3}{|c|}{ Condition } \\
\hline & Read $1 \times$ & Read $3 \times$ & Retrieved \\
\hline \multicolumn{4}{|c|}{ Means } \\
\hline Young & .72 & .83 & $.97(.95)$ \\
\hline Older & .59 & .67 & $.87(.82)$ \\
\hline \multicolumn{4}{|c|}{ Standard Deviations } \\
\hline Young & .13 & .08 & $.03(.04)$ \\
\hline Older & .11 & .11 & $.12(.13)$ \\
\hline
\end{tabular}

Note-Parentheses represent probability of correct recall without conditionalizing on errorless retrieval in the initial study phase.

In the opposition procedure used in Experiment 2 here, increased automaticity produced by retrieval practice would serve as a source of interference. The procedure is outlined in Table 3. The target responses for cue words (e.g., knee-bone) were presented for study in Phase 2, and participants were instructed to recall these for the final test. The first phase of Experiment 2 was the same as that of Experiment 1, except that the responses that were presented and retrieved (e.g., knee-bend) served as competitors for the target responses that were presented in Phase 2. The increased automaticity produced by retrieval practice would result in increased interference from Phase 1 responses (e.g., bend intruding when bone was intended to be recalled).

Experiment 2 differed from the experiments by Jacoby (1999) in that it examined cued recall rather than recognition-memory performance, and it also differed in several other procedural details. Consequently, we did not expect the pattern of results to be identical to that observed by Jacoby (1999). However, we did expect older adults, in comparison with young adults, to be less able to use recollection to oppose the automatic influences of memory resulting from retrieval practice. As support for that prediction, we expected that retrieval practice would increase proactive interference, increasing intrusions of the practiced response more so for older adults than for young adults. That is, we predicted an interaction between retrieval practice (in comparison with repeated reading) and age.

Experiment 2 included a guessing condition (see Table 3) that was identical to the retrieved condition, ex-

Table 3

Illustrative Examples of Conditions in Experiment 2

\begin{tabular}{|c|c|c|c|c|}
\hline Read $1 \times$ & Read $3 \times$ & Retrieved & Guessing & Baseline \\
\hline \multicolumn{5}{|c|}{ Phase 1} \\
\hline sweet-tooth & $\begin{array}{l}\text { judge-jury } \\
\text { judge-jury } \\
\text { judge-jury }\end{array}$ & $\begin{array}{l}\text { knee-bend } \\
\text { knee-b_n_ } \\
\text { knee-b_n_ }\end{array}$ & $\begin{array}{l}\text { rabbit-hole } \\
\text { rabbit-h_e } \\
\text { rabbit-h_ee }\end{array}$ & \\
\hline sweet-taste & judge-just & $\begin{array}{r}\text { Phase } 2 \\
\text { knee-bone }\end{array}$ & & anchor-stop \\
\hline sweet-t_t_ & judge-ju__ & $\begin{array}{l}\text { Final Test } \\
\text { knee-b_n_ }\end{array}$ & rabbit-h_e & anchor-s__ \\
\hline
\end{tabular}

Note-Particular stimuli varied across participants because of counterbalancing. On the final test, the correct response was the word pair from Phase 2, and the intrusion response was the word pair from Phase 1. 
cept that the target response was not studied in Phase 2. This condition was meant to explore the possibility that the retrieval practice effects would be general, so that young adults would show reduced intrusions in comparison with older adults even when the target response had not been presented in the context of the experiment. Alternatively, the benefits of retrieval might not generalize to this condition because of failures to generate a substitute response. For example, a participant might recollect that "rabbit hole" was studied in Phase 1, but then fail to generate "hare" as a substitute. In this case, the intrusion response "hole" might still be given.

One group of young adults and two groups of older adults participated in Experiment 2. The unmatched older adults group followed the same procedure as did the young adults, but showed worse baseline performance, which raised concerns about interpreting results from the retrieved condition. Consequently, a second group of older adults (matched older adults) was given extra study time in Phase 2 in order to match their baseline performance to that of young adults. We expected the age $\times$ retrieval interaction to occur even when baseline performance was matched.

\section{Method}

Participants. There were three groups: young adults, unmatched older adults, and matched older adults (see Table 1). Although the matched group was recruited after the unmatched group, the two older groups were recruited from the same pool and did not differ from each other in terms of age, vocabulary, education, or health (all $p s>.10$ ).

Design, Materials, and Procedure. Only changes relative to Experiment 1 are described here. Five experimental conditions (see Table 3 ) were each represented by 20 word triplets. The assignment of word triplets to conditions was counterbalanced across participants, as was the assignment of response words to be intrusions or targets.

For the final test, participants were instructed to respond with the word presented in Phase 2. Participants were questioned after the final test in order to ensure that they understood the instructions.
Three older adults were excluded and replaced because they were unclear about which phase they were to recall from.

The read- $1 \times$, read- $3 \times$, and retrieved conditions were the same as those in Experiment 1, except that the intrusion response rather than the target was presented in Phase 1. In the guessing condition, retrieval practice of the competing response was provided in Phase 1, but neither the cue nor the target response was presented in Phase 2 . Participants had to guess the correct response on the final test (e.g., "hare"; see Table 3). In the baseline condition, the relevant pair was presented in Phase 2 only, and neither the cue nor the intrusion response was presented in Phase 1.

All pairs presented in Phase 2 were presented once and at a rate of $2 \mathrm{sec}$ for young and unmatched older adults, and a rate of $6 \mathrm{sec}$ for matched older adults.

\section{Results and Discussion}

The probability of producing at least one error for an item during retrieval practice in Phase 1 was lower for young adults $(M=.09)$ than for unmatched and matched older adults $(M \mathrm{~s}=.24$ and .27 , respectively) $[F(2,57)=$ $\left.15.28, p<.00001, \eta^{2}=.35\right]$.

Correct recall. Table 4 shows the probability of correct responses (from Phase 2) and intrusions (from Phase 1). As indicated by symbols in the table, matching was successful in that $t$ tests showed no difference between matched older adults and young adults on baseline correct recall. In contrast, unmatched older adults performed worse than the young.

Importantly, aging was associated with less correct recall in the retrieved condition than in the read- $3 \times$ condition, and this was true for both older adult groups. An ANOVA was performed on correct responses in all five conditions $\times$ all three groups in order to ensure that the overall interaction was significant (which it was), with the Greenhouse-Geisser correction for violated sphericity $\left[F(6.8,194.8)=3.34, p<.01, \eta_{\mathrm{p}}^{2}=.10\right]$. Next, a more restricted $2 \times 2$ ANOVA compared young and unmatched

Table 4

Means and Standard Deviations of the Probability of Correct Recall and Intrusions in Experiment 2

\begin{tabular}{lcccccc}
\hline \multicolumn{1}{c}{ Age } & Read 1 & Read $3 \times$ & Retrieved & Guessing & Baseline \\
\hline \multicolumn{7}{c}{ Mean Correct Recall } \\
Young & .50 & .47 & .52 & $(.53)$ & $.14(.15)$ & .67 \\
Older unmatched & .46 & $.37^{*}$ & $.28^{* * *}(.30)^{* * *}$ & $.08(.13)$ & $.58^{*}$ \\
Older matched & .51 & .42 & $.33^{* *}$ & $(.36)^{* *}$ & $.10(.14)$ & .67 \\
& \multicolumn{7}{c}{ Mean Intrusions } \\
Young & .42 & .47 & .45 & $(.44)$ & $.80(.79)$ & .19 \\
Older unmatched & .44 & .53 & $.69^{* *}(.64)^{* *}$ & $.87(.77)$ & .22 \\
Older matched & .41 & .50 & $.61^{*}(.57)^{*}$ & $.85(.78)$ & .19 \\
& Standard Deviations of Correct Recall & & \\
Young & .16 & .14 & .24 & $(.23)$ & $.13(.14)$ & .14 \\
Older unmatched & .11 & .11 & .16 & $(.15)$ & $.06(.09)$ & .12 \\
Older matched & .12 & .16 & .17 & $(.15)$ & $.06(.09)$ & .11 \\
& \multicolumn{7}{c}{ Standard Deviations of Intrusions } \\
Young & .14 & .16 & .25 & $(.24)$ & $.18(.18)$ & .11 \\
Older unmatched & .10 & .12 & .17 & $(.17)$ & $.09(.14)$ & .08 \\
Older matched & .11 & .15 & .16 & $(.17)$ & $.09(.12)$ & .07 \\
\hline
\end{tabular}

Note-Parentheses represent probabilities without conditionalizing on errorless retrieval in the initial study phase. Asterisks indicate significant differences compared with the corresponding mean from younger adults. ${ }^{*} p<.05 .{ }^{* *} p<.01 .{ }^{* * *} p<.001$. 
older adults in the read $-3 \times$ and retrieved conditions in order to reveal a significant interaction $[F(1,38)=6.47$, $\left.p<.05, \eta_{\mathrm{p}}^{2}=.15\right]$. Finally, a $2 \times 2$ ANOVA also revealed a significant interaction when comparing young and matched older adults in the read- $3 \times$ and retrieved conditions $\left[F(1,38)=5.26, p<.05, \eta_{\mathrm{p}}^{2}=.12\right]$.

Intrusions. Analyses of intrusions suggest that the poor performance of older adults in the retrieved condition was due to increased intrusions of the earlier retrieved responses. First, the overall $5 \times 3$ ANOVA revealed a significant interaction, with the Greenhouse-Geisser correction $\left[F(6.8,194.7)=2.86, p<.01, \eta_{\mathrm{p}}^{2}=.09\right]$. Next, a $2 \times 2$ ANOVA showed a significant interaction when comparing young and unmatched older adults in the read- $3 \times$ and retrieved conditions $\left[F(1,38)=7.65, p<.01, \eta_{\mathrm{p}}^{2}=.17\right]$. Finally, a $2 \times 2$ ANOVA also showed a significant interaction when comparing young and matched older adults in the read- $3 \times$ and retrieved conditions $[F(1,38)=4.89$, $\left.p<.05, \eta_{\mathrm{p}}^{2}=.11\right] .^{1}$

Similar to what was found for study repetition in recognition memory (Jacoby, 1999), retrieval practice had separate effects on controlled and automatic influences in cued recall. Retrieval practice had the automatic influence of increasing the accessibility of the practiced response, which could serve as a source of interference. For young adults, retrieval practice also enhanced controlled recollection, which was used to successfully oppose this increased automatic influence. However, because of older adults' lessened ability to recollect, the automatic, interfering influence of retrieval practice was largely unopposed. The age difference in interfering effects of retrieval practice was observed even when older adults were equated to young adults in a baseline condition.

There was a trend in the means suggesting that young adults had fewer intrusions in the guessing condition as well. However, the effect of group was not significant there, as shown by a one-way ANOVA $[F(2,57)=1.67$, $p=.20]$. It is possible that even when recollection succeeded, participants often gave the intrusion response because it was difficult to generate a suitable substitute when none had been presented in the context of the experiment. Indeed, it was rare for suitable substitutes to be reported in the guessing condition (this is labeled Correct Recall in Table 4). Age differences in retrieval effects might be most apparent when at least two appropriate responses are readily available.

Neither repetition nor retrieval practice caused a significant change in intrusions in young adults' cued-recall performance. In contrast, Jacoby (1999) found that repetition caused a decrease in false alarms in recognition-memory performance. Different results are unsurprising, given the different measures of memory and other procedural differences. Repetition or retrieval would be expected to cause a significant decrease in interference only if the effects on recollection were larger than those on automatic influences of memory. More research is needed in order to uncover the factors that are responsible for the balance of the two effects in both repetition and spaced retrieval.
Importantly, the pattern of results across experiments does suggest that spaced retrieval had two effects for young adults rather than no effect. In Experiment 1, spaced retrieval did enhance memory performance for young adults. Given this result, it would be difficult to explain the age $\times$ retrieval interaction in Experiment 2 without postulating opposing processes along with age differences in recollection.

\section{GENERAL DISCUSSION}

The results of Experiment 1 showed that spaced-retrieval practice enhanced later memory performance more than did additional study. The results of Experiment 2 indicated that spaced retrieval had the automatic effect of increasing the accessibility of practiced responses, and the second effect of enhancing controlled recollection. The balance of these two effects places important limitations on the utility of retrieval practice as a means of improving memory. When a person's ability to recollect is impaired, the facilitation gained by increasing the accessibility of correct responses (Experiment 1) is offset by the cost when those highly accessible responses are incorrect (Experiment 2).

We have argued that retrieval enhances two processes, although we have not explained why retrieval would enhance either. Carrier and Pashler (1992) suggested that retrieval benefits can potentially be explained by network models that learn through error-reduction mechanisms. In such models, an external presentation of a response (such as that in the read- $3 \times$ condition) can spoil the network's ability to generate its own noisy representation and then learn from it. With this in mind, the most promising network models of retrieval effects on memory might be those that include error-correction learning mechanisms within a dual-process or dual-system framework (see, e.g., O’Reilly \& Rudy, 2000).

Although the present results were framed in terms of a recollection impairment in older adults, note that the results are also consistent with age-related impairments in other varieties of controlled processing, such as source memory (Benjamin \& Craik, 2001) or goal neglect (Jacoby, Bishara, Hessels, \& Toth, 2005). Additional research is required to choose among more fine-grained distinctions regarding controlled processing. Overall, the present results suggest that older adults and perhaps other populations with controlled memory impairments might suffer from inflexible behavior following spaced retrieval practice. This idea should be kept in mind when spaced retrieval is being considered as a tool for memory rehabilitation.

\section{AUTHOR NOTE}

We thank Danielle Hirschfeld, Carole Jacoby, Alex Konkel, Meredith Reuter, Chad Rogers, Michael Schachter, and Aaron Shumaker for helping with data collection, data coding, and other aspects of this research. This research was supported by Grant AG13845 from the National Institute on Aging. Correspondence should be sent to A. J. Bishara, Indiana University, Department of Psychological \& Brain Sciences, 1101 East Tenth Street, Bloomington, IN 47405 (e-mail: abishara@indiana.edu). 


\section{REFERENCES}

Benjamin, A. S., \& CRAIK, F. I. M. (2001). Parallel effects of aging and time pressure on memory for source: Evidence from the spacing effect. Memory \& Cognition, 29, 691-697.

Bird, M., \& Kinsella, G. (1996). Long-term cued recall of tasks in senile dementia. Psychology \& Aging, 11, 45-56.

Camp, C. J., Foss, J. W., O'Hanlon, A. M., \& Stevens, A. B. (1996). Memory interventions for persons with dementia. Applied Cognitive Psychology, 10, 193-210.

CARrier, M., \& PAshler, H. (1992). The influence of retrieval on retention. Memory \& Cognition, 20, 633-642.

Cherry, K. E., Simmons, S. S., \& Camp, C. J. (1999). Spaced retrieval enhances memory in older adults with probable Alzheimer's disease. Journal of Clinical Geropsychology, 5, 159-175.

Glisky, E. L., Schacter, D. L., \& Tulving, E. (1986). Learning and retention of computer-related vocabulary in memory-impaired patients: Method of vanishing cues. Journal of Clinical \& Experimental Neuropsychology, 8, 292-312.

JACOBY, L. L. (1996). Dissociating automatic and consciously controlled effects of study/test compatibility. Journal of Memory \& Language, 35, 32-52.

JACOBY, L. L. (1999). Ironic effects of repetition: Measuring age-related differences in memory. Journal of Experimental Psychology: Learning, Memory, \& Cognition, 25, 3-22.

JACOBY, L. L., \& BARTZ, W. H. (1972). Rehearsal and transfer to LTM. Journal of Verbal Learning \& Verbal Behavior, 11, 561-565.

Jacoby, L. L., Bishara, A. J., Hessels, S., \& Toth, J. P. (2005). Aging, subjective experience, and cognitive control: Dramatic false remembering by older adults. Journal of Experimental Psychology: General, 134, 131-148.

JENNINGS, J. M., \& JACOBY, L. L. (1993). Automatic versus intentional uses of memory: Aging, attention, and control. Psychology \& Aging, 8, 283-293.

JeNnings, J. M., \& JACOBY, L. L. (1997). An opposition procedure for detecting age-related deficits in recollection: Telling effects of repetition. Psychology \& Aging, 12, 352-361.

Kapur, N., Glisky, E. L., \& Wilson, B. A. (2004). Technological memory aids for people with memory deficits. Neuropsychological Rehabilitation, 14, 41-60.

LANDAUER, T. K., \& BJoRK, R. A. (1978). Optimal rehearsal patterns and name learning. In M. M. Gruneberg, P. E. Harris, \& R. N. Sykes (Eds.), Practical aspects of memory (pp. 625-632). New York: Academic Press.

LogAN, J. M., \& BALOTA, D. A. (in press). Expanded vs. equal interval spaced retrieval practice: Exploring different schedules of spacing and retention interval in younger and older adults. Aging, Neuropsychology, \& Cognition.

Nelson, D. L., McEvoy, C. L., \& Schreiber, T. A. (1998). The University of South Florida word association, rhyme, and word fragment norms. Retrieved October 1, 2002, from w3.usf.edu/FreeAssociation/.

O'ReILly, R. C., \& RUdY, J. W. (2000). Computational principles of learning in the neocortex and hippocampus. Hippocampus, 10, 389-397.

RoEDiger, H. L., III, \& KARPICKE, J. D. (2006). Test-enhanced learning: Taking memory tests improves long-term retention. Psychological Science, 17, 249-255.

Schacter, D. L., Rich, S. A., \& Stampp, M. S. (1985). Remediation of memory disorders: Experimental evaluation of the spaced-retrieval technique. Journal of Clinical \& Experimental Neuropsychology, 7, 79-96.

\section{NOTE}

1. In Experiment 2, the 2 (young vs. matched older adults) $\times 2$ (read $3 \times$ vs. retrieved) ANOVA on intrusion responses showed only a marginally significant interaction when the data were not conditionalized on errorless retrieval in Phase $1\left[F(1,38)=3.57, p<.07, \eta_{\mathrm{p}}^{2}=.09\right]$.

(Manuscript received June 22, 2006; revision accepted for publication May 4, 2007.) 